\title{
Bacterial Etiology of Bloodstream Infections and Antimicrobial Resistance Patterns from a Tertiary Care Hospital in Malé, Maldives
}

\author{
Aishath Maharath (iD ${ }^{1}$ and Mariyam Shabeena Ahmed ${ }^{2}$ \\ ${ }^{1}$ Faculty of Health Sciences, The Maldives National University, Malé 20371, Maldives \\ ${ }^{2}$ Research Center, The Maldives National University, Malé 20371, Maldives \\ Correspondence should be addressed to Aishath Maharath; aishath.maharath@mnu.edu.mv
}

Received 23 June 2021; Accepted 1 October 2021; Published 18 October 2021

Academic Editor: Todd R. Callaway

Copyright (C) 2021 Aishath Maharath and Mariyam Shabeena Ahmed. This is an open access article distributed under the Creative Commons Attribution License, which permits unrestricted use, distribution, and reproduction in any medium, provided the original work is properly cited.

\begin{abstract}
Background. Bloodstream infections pose a significant health problem worldwide and is a major cause of morbidity and mortality in many countries. It is important to have country-specific data for major pathogens causing bloodstream infections, in light of emerging resistance patterns of common bacterial isolates. Due to the scarcity of reports in this area, the aim of this study was to identify bacterial pathogens causing bloodstream infections among the study population. Methods. A retrospective analysis of blood culture samples received at the Department of Laboratory Medicine, Indira Gandhi Memorial Hospital, Malé, Maldives, was performed for reports between January 2016 and December 2017. Results. Out of the 471 culture-positive samples, 278 (59\%) were males and 193 (41\%) were females. Amongst the culture-positive samples, 338 (71.8\%) Gram-positive organisms were isolated and 133 (28.2\%) Gram-negative organisms were isolated. Coagulase-negative Staphylococcus (CoNS) was the most frequently isolated blood-borne bacterial pathogen in this study, accounting for $53.6 \%$ and $50.9 \%$ of the isolates in 2016 and 2017, respectively. Other frequently isolated pathogens included Staphylococcus aureus (15.9\% and 10.3\%), Klebsiella spp. (10.5\% and 16.4\%), and Escherichia coli (7.1\% and 10.8\%). Coagulase-negative Staphylococcus (CoNS) revealed high percentage of resistance among the tested antimicrobials, ampicillin, cephalexin, cefotaxime, and gentamicin. Over the two years, a significant difference between the percentage resistance among paediatric and adult patients was observed for coagulase-negative Staphylococcus (CoNS) isolate resistance to ampicillin $(p \leq 0.001)$, cephalexin $(p \leq 0.001)$, cefotaxime $(p \leq 0.001)$, gentamicin $(p=0.008)$, and cotrimoxazole (SXT) $(p \leq 0.001)$. When comparing the significant antimicrobial resistance trends, it can be seen that Enterobacteriaceae isolates also demonstrated high resistance to ampicillin and gentamicin as well as second- and third-generation cephalosporins. Conclusions. This study highlights the major bacterial pathogens involved in bloodstream infections in the healthcare setting of Malé, Maldives, and antibiotic resistance patterns. The results indicate that further characterization of bacteremia and its resistance patterns is needed to combat bloodstream infections.
\end{abstract}

\section{Introduction}

Bloodstream infections (BSI) are defined broadly as the presence of viable microorganisms in the blood, which can lead to inflammation in the host and alter the clinical and hemodynamic properties and lead to morbid consequences [1]. The presence of microorganisms, however, transiently in the circulation poses a threat to most organs. The consequences of bloodstream infections if not treated can lead to shock, disseminated intravascular coagulation (DIC), multiple organ failure, and death [2]. Bloodstream infections are a major public health problem worldwide, and it has been associated with significant morbidity and mortality [3]. Although it is still common in developed nations, the burden is high in the least developed and developing countries [4].

There is a considerable variation in epidemiology and pathogen profile of microorganisms, which cause BSI [5]. Population-based studies originating in countries such as 
Australia, Canada, Denmark, Finland, Iceland, New Zealand, Sweden, and USA show the etiologies of BSI to be mainly from the organisms Escherichia coli, Staphylococcus aureus, and Streptococcus pneumoniae [3]. In contrast to this, the pathogen profiles vary in Africa and Asia. Salmonella enterica has been implicated as one of the major pathogens causing BSI in both African and Asian nations $[6]$.

The potential outcomes of BSI and the delays in performing and receiving culture results often lead to empirical treatment. In developing countries, this may also be due to the lack of treatment guidelines and unavailability of susceptibility patterns for local isolates [3]. Even in constrained healthcare settings, the knowledge of local antibiotic resistance patterns will help in selecting effective empirical therapy [7]. As such there is an emerging trend of BSI caused by Gram-negative organisms and an increased incidence of drug-resistant strains [8].

In light of the growing trends of BSI infections globally and emerging antimicrobial resistance profiles of implicated organisms, it is important to conduct studies to investigate the pathogen profiles for Maldives. In addition to this, published data for the country is limited and there is a need for baseline studies in this area. Therefore, a retrospective study was conducted to determine the common bacterial agents associated with bloodstream infections and their antibiotic resistance patterns.

\section{Methods}

In this retrospective study, blood culture sample results were obtained for both out- and inpatients who attended the Indira Gandhi Memorial Hospital, which is a tertiary hospital located in Malé, the capital city of the Republic of Maldives. This is a 350-bed government hospital which is located in the population dense capital island. A total of 9556 blood sample results from January 2016 to December 2017 were processed. Oxoid SIGNAL (Thermo Fisher Scientific Inc.) blood culture systems were used to identify the presence of pathogens.

Collected blood culture samples were inoculated into Oxoid SIGNAL (Thermo Fisher Scientific Inc.) blood culture system and incubated at $37^{\circ} \mathrm{C}$ for 7 days or until the presence of a pathogen is indicated from the Oxoid SIGNAL blood culture system. Positive culture samples were directly inoculated into MacConkey agar and blood agar plates. The plates were incubated aerobically at $37^{\circ} \mathrm{C}$ and examined after 18-24 hrs. Bacterial organisms were isolated using standard bacteriological procedures based on the current Clinical and Laboratory Standards Institute (CLSI) 2014-2017 guideline.

Antimicrobial susceptibility tests were carried out by using the disk diffusion method (modified Kirby-Bauer method) on Mueller-Hinton agar (HiMedia Laboratories, India). Antibiotics that were used in this study include ampicillin $(10 \mu \mathrm{g})$, ampicillin-sulbactam $(10 / 10 \mu \mathrm{g})$, amoxicillin-clavulanate $(20 / 10 \mu \mathrm{g})$, piperacillin-tazobactam (100/ $10 \mu \mathrm{g})$, cephalexin $(30 \mu \mathrm{g})$, cefuroxime $(30 \mu \mathrm{g})$, cefotaxime $(30 \mu \mathrm{g})$, ceftazidime $(30 \mu \mathrm{g})$, ceftriaxone $(30 \mu \mathrm{g})$, cefepime $(30 \mu \mathrm{g})$, imipenem $(10 \mu \mathrm{g})$, meropenem $(10 \mu \mathrm{g})$, gentamicin
$(10 \mu \mathrm{g})$, tobramycin $(10 \mu \mathrm{g})$, amikacin $(30 \mu \mathrm{g})$, netilmicin $(30 \mu \mathrm{g})$, ciprofloxacin $(5 \mu \mathrm{g})$, cotrimoxazole $(1.25 / 23.75 \mu \mathrm{g})$, and colistin $(30 \mu \mathrm{g})$ from HiMedia Laboratories, India. The ATCC cultures Staphylococcus aureus ATCC 25923 and Escherichia coli ATCC 25218 were used as control strains.

In this study, multidrug resistance (MDR) was categorized as acquired nonsusceptibility to at least three classes of antibiotics [9].

2.1. Ethical Considerations. Ethical clearance was obtained from the Maldives National University as well as National Health Research Committee, Ministry of Health, Malé, Maldives. Patient data was kept anonymous throughout the study and kept confidential.

2.2. Statistical Analysis. Culture positivity was seen in 471(4.9\%) samples, and 9085(95.1\%) samples were negative. The 471 culture positive samples were entered into the Statistical Package for Social Sciences (SPSS) version 20.0, and all the analysis was carried out using this program.

The data were analyzed using chi-square $\left(\chi^{2}\right)$, independent samples $t$-test, and frequency distributions. $\chi^{2}$ was conducted to find out the significant difference between the bacterial isolates related to age groups over the two years. $t$-test was carried out to find out the significant difference between the percentage resistance related to individual microbials for each bacterial isolate. These analyses were carried out with $95 \%$ confidence interval (CI) and a $p$ value less than 0.05 was regarded as significant. The frequency distribution was used to compare the difference in BSI among male, female, and age groups over the years.

\section{Results}

Out of the 471 culture-positive samples, 278 (59\%) were males and 193 (41\%) were females. Amongst the culturepositive samples, 338 (71.8\%) Gram-positive organism were isolated and 133 (28.2\%) Gram-negative organisms were isolated. The data contained specimens obtained from paediatric patients $(n=130,27.6 \%)$ and adult patients ( $n=341,72.4 \%)$ (Table 1$)$.

Table 2 shows the percentage distribution of organism isolated from blood culture samples during the two-year study period. Coagulase-negative Staphylococcus (CoNS) was the most frequently isolated blood-borne bacterial pathogen in this study. Other frequently isolated pathogens included Staphylococcus aureus, Klebsiella spp., and Escherichia coli.

As illustrated in Table 3, Gram-positive bacteria (71.8\%) were the most common pathogenic agents compared to the Gram-negative bacteria $(28.2 \%)$ in this study $\left(\chi^{2}=5.533 ; p=0.019\right)$. Table 4 details the common bacterial isolates causing BSI among the two group of patients. Predominant isolates among Gram-positive isolates were coagulase-negative Staphylococcus (CoNS) 246 (52.2\%), Staphylococcus aureus 62 (13.2\%), Streptococcus spp. 23 (4.9\%), and Enterococcus spp. 7 (1.5\%). Among the Gramnegative isolates, the most predominant was Klebsiella spp. 
TABLE 1: Trends in the organisms isolated from the culture-positive patient samples.

\begin{tabular}{lccccc}
\hline \multirow{2}{*}{ Organisms } & \multirow{2}{*}{ Bacterial isolate $(\%)$} & Paediatric $(n=130)$ & \multicolumn{2}{c}{ Adult $(n=341)$} \\
& & Male $(n=80)$ & Female $(n=50)$ & Male $(n=198)$ & Female $(n=143)$ \\
\hline Gram-positive & $338(71.8)$ & $68(85.0)$ & $39(78.0)$ & $136(68.7)$ & $95(66.4)$ \\
Gram-negative & $133(28.2)$ & $12(15.0)$ & $11(22)$ & $62(31.3)$ & $48(33.6)$ \\
\hline
\end{tabular}

TABLE 2: Distribution of organism isolated from blood culture samples during the two years.

\begin{tabular}{lccc}
\hline Bacterial isolates & Number (\%) & Year & 2016 \\
\hline Streptococcus spp. & $23(4.9)$ & $14(5.9)$ & $9(3.9)$ \\
Coagulase-negative Staphylococcus (CoNS) & $246(52.2)$ & $128(53.6)$ & $118(50.9)$ \\
Staphylococcus aureus & $62(13.2)$ & $38(15.9)$ & $24(10.3)$ \\
Enterococcus spp. & $7(1.5)$ & $3(1.3)$ & $4(1.7)$ \\
Klebsiella spp. & $63(13.4)$ & $25(10.5)$ & $3(1.7)$ \\
Acinetobacter spp. & $7(1.5)$ & $3(1.3)$ & $3(1.3)$ \\
Pseudomonas aeruginosa & $5(1.1)$ & $4(1.7)$ & $2(0.9)$ \\
Pseudomonas spp. & $12(2.5)$ & $17(7.1)$ & $8(3.4)$ \\
Escherichia coli & $42(8.9)$ & $3(1.3)$ & $25(10.8)$ \\
Salmonella spp. & $4(0.8)$ & 239 & $1(0.4)$ \\
Total & 471 & & 232 \\
\hline
\end{tabular}

TABLE 3: Trends of Gram-positive and Gram-negative bacterial isolates.

\begin{tabular}{|c|c|c|c|c|c|}
\hline & Number (\%) & $2016(n=239)$ & $2017(n=232)$ & $\chi^{2}$ & $p$ value \\
\hline Gram-positive & $338(71.8)$ & $183(76.6)$ & $155(66.8)$ & \multirow{2}{*}{5.533} & \multirow{2}{*}{0.019} \\
\hline Gram-negative & $133(28.2)$ & $56(23.4)$ & $77(33.2)$ & & \\
\hline
\end{tabular}

TABLE 4: Trends of bacterial isolates among paediatric and adult populations, $n=(471)$.

\begin{tabular}{|c|c|c|c|c|c|c|}
\hline \multirow{2}{*}{ Bacterial isolates } & \multirow{2}{*}{ Number (\%) } & \multicolumn{2}{|c|}{ Paediatric patients $(n=130)$} & \multicolumn{2}{|c|}{ Adult patients $(n=341)$} & \multirow{2}{*}{$p$ value } \\
\hline & & $2016(n=75$ & $2017(n=55$ & $2016(n=164$ & $2017(n=177$ & \\
\hline Gram-positive isolates & $338(71.8 \%)$ & $61(81.3)$ & $46(83.6)$ & $122(74.4)$ & $109(61.6)$ & \\
\hline Streptococcus spp. & $23(4.9)$ & $8(10.7)$ & $5(9.1)$ & $6(3.7)$ & $4(2.3)$ & 0.940 \\
\hline Coagulase-negative Staphylococcus (CoNS) & $246(52.2)$ & $49(65.3)$ & $35(63.6)$ & $79(48.2)$ & $83(46.9)$ & 0.154 \\
\hline Staphylococcus aureus & $62(13.2)$ & $3(4.0)$ & $6(10.9)$ & $35(21.3)$ & $18(10.2)$ & 0.063 \\
\hline Enterococcus spp. & $7(1.5)$ & $1(1.3)$ & $0(0.0)$ & $2(1.2)$ & $4(2.3)$ & 0.212 \\
\hline Gram-negative isolates & $133(28.2)$ & $14(18.7)$ & $9(16.4)$ & $42(25.6)$ & $68(38.4)$ & \\
\hline Klebsiella spp. & $63(13.4)$ & $6(8.0)$ & $6(10.9)$ & $19(11.6)$ & $32(18.1)$ & 0.417 \\
\hline Acinetobacter spp. & $7(1.5)$ & $4(5.3)$ & $3(5.5)$ & $4(2.4)$ & $3(1.7)$ & - \\
\hline Pseudomonas aeruginosa & $5(1.1)$ & $1(1.3)$ & $0(0.0)$ & $2(1.2)$ & $2(1.1)$ & 0.361 \\
\hline Pseudomonas spp. & $12(2.5)$ & $3(4.0)$ & $1(1.8)$ & $1(0.6)$ & $7(4.0)$ & 0.030 \\
\hline Escherichia coli & $42(8.9)$ & $3(4.0)$ & $1(1.8)$ & $14(8.5)$ & $24(13.6)$ & 0.139 \\
\hline Salmonella spp. & $4(0.8)$ & $1(1.3)$ & $1(1.8)$ & $2(1.2)$ & $0(0.0)$ & 0.248 \\
\hline
\end{tabular}

63 (13.4\%) followed by Escherichia coli 42 (8.9\%), Pseudomonas spp. 12 (2.5\%), Acinetobacter spp. 7(1.5\%), Pseudomonas aeruginosa 5 (1.1\%), and Salmonella spp. 4 (0.8\%).

Based on the age categories, the data contained specimens obtained from neonates $(n=71)$, infants $(n=21)$, children $(n=38)$, adults $(n=163)$, and elderly $(n=176)$ (Table 4). During the two years of data collection, more adult patients were found with BSI $(72.4 \%)$ as compared to paediatric patients $(27.6 \%)$. But this trend was not statistically significant $(p=0.063)$ (Table 5$)$.

When compared between the two years, a significant difference is seen in adults (31.8\%, 38.4\%; $p=0.017)$. Among the paediatric group, neonates comprised a high proportion of culture positive samples $(15.1 \%)$ in comparison to infants $(4.5 \%)$ and children $(8.1 \%)$. Elderly comprised dominant culture positive samples (37.4\%) in comparison to adults (34.6\%). Amongst all cases, the highest proportion of positive BSI was seen among the elderly (37.4\%) (Table 6).

When considering the trends of antimicrobial susceptibilities of Gram-positive isolates in the study, coagulasenegative Staphylococcus (CoNS) revealed high level of resistance among tested antimicrobials over the two years (Table 7). Over the two years, a significant difference between the percentage resistance among paediatric and adult patients was observed for coagulase-negative Staphylococcus 
TABLE 5: Trends of group of patients associated with BSI.

\begin{tabular}{lcccc}
\hline Patient group & Number $(\%)$ & $2016(n=239)$ & $2017(n=232)$ & $\chi^{2}$ \\
\hline Paediatric & $130(27.6)$ & $75(31.4)$ & $55(23.7)$ & \multirow{2}{*}{3.469} \\
Adult & $341(72.4)$ & $164(68.7)$ & $177(76.2)$ & 0.063 \\
\hline
\end{tabular}

TABLE 6: Trends of BSI among various group of patients

\begin{tabular}{lcccccc}
\hline Patient group & Age category & Age group & Number $(\%)$ & $2016(n=239)$ & $2017(n=232)$ & $p$ value \\
\hline \multirow{3}{*}{ Paediatrics } & Neonates & $<28$ days & $71(15.1)$ & $44(18.4)$ & $27(11.6)$ & 0.549 \\
& Infants & $>28$ days to 1 year & $21(4.5)$ & $11(4.6)$ & $10(4.3)$ & 0.223 \\
& Children & $1-15$ years & $38(8.1)$ & $20(8.4)$ & $18(7.8)$ & 0.239 \\
\hline \multirow{2}{*}{ Adults } & Adults & $16-65$ years & $163(34.6)$ & $76(31.8)$ & $89(38.4)$ & $\mathbf{0 . 0 1 7}$ \\
& Elderly & $>65$ years & $176(37.4)$ & $88(36.9)$ & $88(37.9)$ & 0.589 \\
\hline
\end{tabular}

Note: $p$ values in bold indicate significant effects.

(CoNS) isolate resistance to ampicillin $(p \leq 0.001)$, cephalexin $(p \leq 0.001)$, cefotaxime $(p \leq 0.001)$, gentamicin $(p=0.008)$, and cotrimoxazole (SXT) $(p \leq 0.001)$. Staphylococcus aureus has also shown significant difference in the percentage resistance among the two group of patients to ampicillin $(p \leq 0.001)$, cephalexin $(p \leq 0.001)$, and ciprofloxacin $(p=0.005)$.

Both Tables 8 and 9 show the trend of antimicrobial resistance in Gram-positive and Gram-negative isolates over the two years of study period, respectively. Among Gramnegative isolates in the study, Klebsiella spp. revealed high level of resistance among tested antimicrobials over the two years (Table 9). Over the two years, a significant difference was observed for Klebsiella spp. resistance to ampicillin ( $p \leq 0.001)$, ampicillin/sulbactam $(p \leq 0.001)$, piperacillin/ tazobactam $(p \leq 0.001)$, cephalexin $(p=0.025)$, cefuroxime $(p=0.004), \quad$ cefotaxime $\quad(p=0.032), \quad$ ceftriaxone $(p=0.010)$, cefepime $(p \leq 0.001)$, and ciprofloxacin $(p=$ 0.034 ) (Table 9). Escherichia coli isolates showed significant resistance to antimicrobials ampicillin $(p=0.001)$, cephalexin $(p=0.022)$, cefuroxime $(p=0.018)$, cefotaxime $(p=0.005), \quad$ ceftazidime $\quad(p=0.041), \quad$ ceftriaxone $(p=0.046)$, and ciprofloxacin $(p=0.038)$. Similarly, Acinetobacter spp. showed significant resistance to cephalosporins cefotaxime $(p=0.010)$, ceftriaxone $(p=0.010)$, and aminoglycoside gentamicin $(p=0.010)$ (as well as fluoroquinolone and ciprofloxacin $(p=0.010)$ ).

Trends of multidrug-resistant (MDR) isolates showed that coagulase-negative Staphylococcus (CoNS) was comparatively higher at $45.2 \%$ (Table 10). Klebsiella spp. at $31.3 \%$ MDR isolates had a statistically significant increase when compared between the two years of study.

\section{Discussion}

The data showed a variation in bloodstream infections (BSI) between age groups. The elderly comprised the dominant culture-positive group (37.4\%) in comparison to the adult population. Neonates comprised a high proportion of culture positive cases (15.1\%) amongst the paediatric group. It is interesting to see culture-positive samples in extremes of ages, and the reason for this distribution is not clear as risk factors for development of blood culture infection have not been evaluated in this particular study.

Studies have implicated a higher risk for neonates for central line-associated bloodstream infections, which comprise a significant component of healthcare-associated infections [10]. This may be due to host risk factors mainly relative to immunodeficiency and breach of physical barriers or due to contamination during access of the catheters [11]. The major organisms implicated in central line-associated bloodstream infections are routine skin colonizers, namely, coagulase-negative Staphylococcus (CoNS), Staphylococcus aureus, and Candida species [12].

In this study,71.8\% of bloodstream infections were caused by Gram-positive bacteria and $28.2 \%$ by Gramnegative bacteria. Similar findings were seen from studies by Shrestha et al., Nepal [13], Arora et al., India [14], Moyo et al., Tanzania [15], and Takeshita et al., Vietnam [16]. In contrast to this, Gram-negative bacteria have been implicated as the commonest cause of BSI in studies by Parajuli et al., Nepal [17], Khurana et al., India [18], Dramowski et al., South Africa [19], and Easow et al., Nepal [20].

Among the Gram-positive organisms, coagulase-negative Staphylococcus (CoNS) (52.2\%) was the most common bacterial pathogen causing BSI in this study. Although there was no statistically significant variation between the paediatric and adult populations, paediatric isolates should be dealt with care and there is a need to differentiate between contaminants and true pathogens. Although coagulasenegative Staphylococcus (CoNS) was noted as contaminants in the past [21], studies have shown that coagulase-negative Staphylococcus (CoNS) and viridans group Streptococcus are frequently associated with immunocompromised paediatric bloodstream infections [22, 23]. Numerous studies have been carried out on the process of ruling out contaminants from pathogens among coagulase-negative Staphylococus $[24,25]$. Studies comparing low- and middle-income countries amongst Asia and Africa regions have showed variation in the causative organisms implicated in BSI in paediatric populations $[26,27]$. Meta-analysis by Droz et al. [28] showed that Gram-negative bacteria accounted for $63.9 \%$ of BSI and Salmonella spp. was the most common pathogen in Asia, while $S$. aureus and S. pneumoniae were 


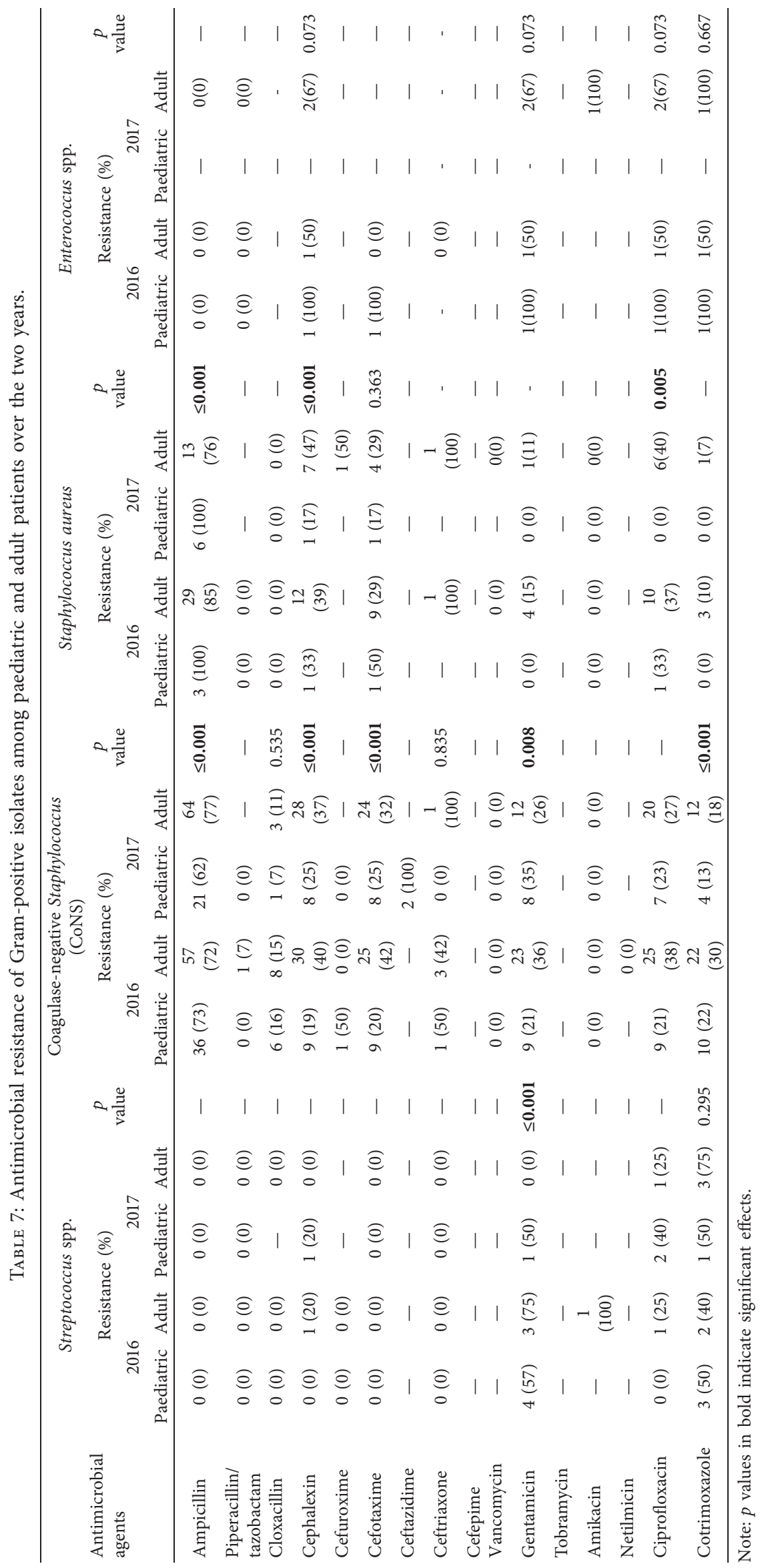




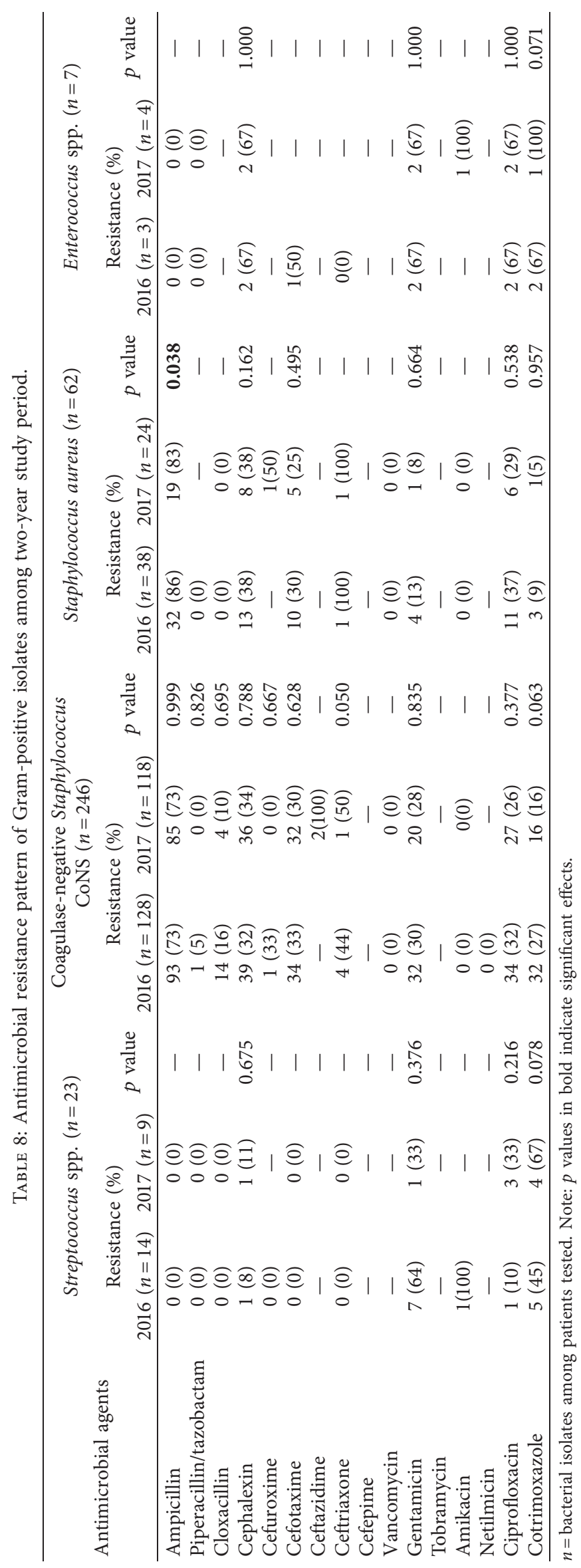




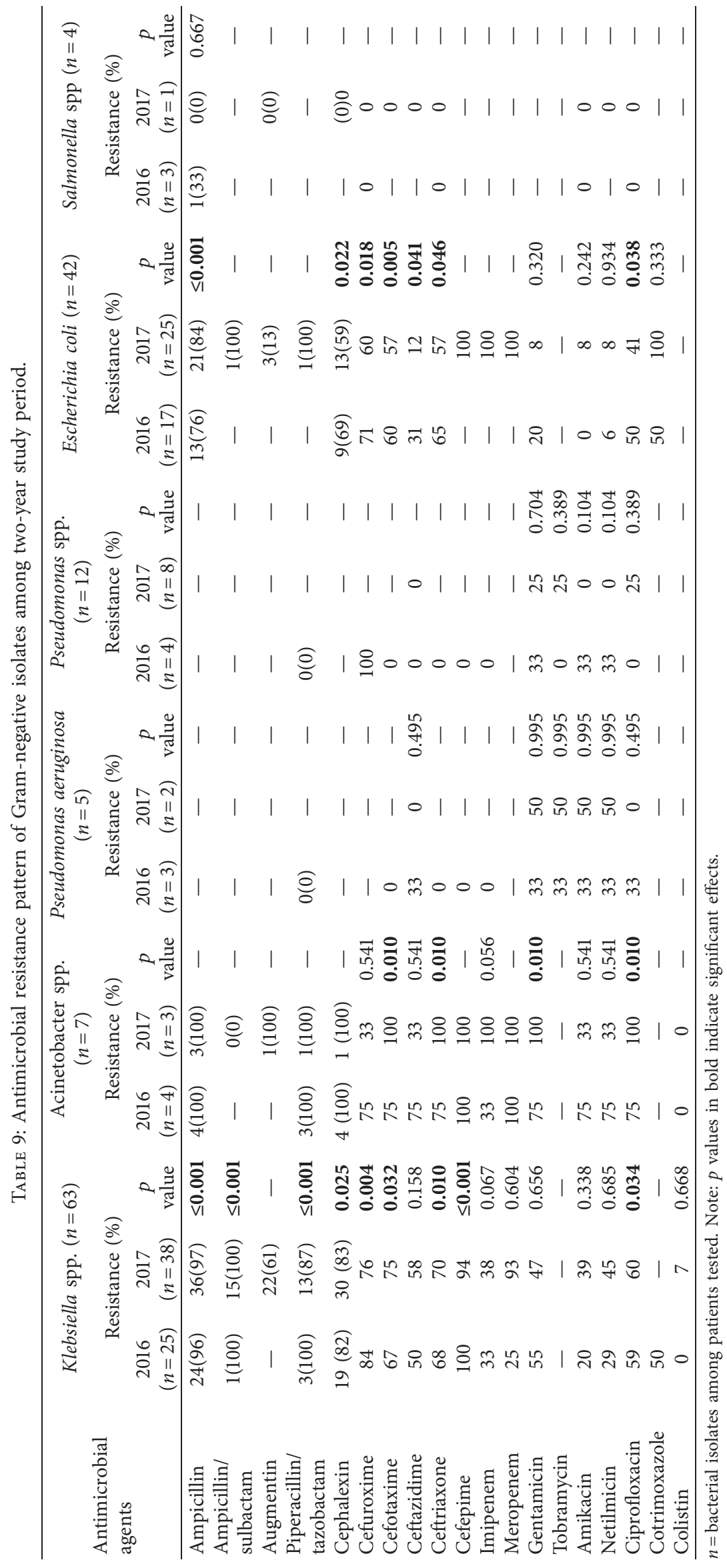


TABLE 10: Multidrug resistance (MDR) isolates for the two-year study period.

\begin{tabular}{|c|c|c|c|c|}
\hline \multirow{2}{*}{ Bacterial isolates } & \multirow{2}{*}{ Number (\%) } & \multicolumn{2}{|c|}{ Year } & \multirow{2}{*}{$p$ value } \\
\hline & & $2016 n=239$ & $2017 n=232$ & \\
\hline Coagulase-negative Staphylococcus (CoNS) & $62(45.2)$ & $37(52.1)$ & $25(37.8)$ & $\leq 0.001$ \\
\hline Staphylococcus aureus & $10(7.2)$ & $8(11.2)$ & $2(3.0)$ & $\leq \mathbf{0 . 0 0 1}$ \\
\hline Enterococcus spp. & $4(3.0)$ & $2(2.8)$ & $2(3.0)$ & 0.182 \\
\hline Klebsiella spp. & $43(31.3)$ & $15(21.1)$ & $28(42.4)$ & $\leq \mathbf{0 . 0 0 1}$ \\
\hline Acinetobacter spp. & $4(3.0)$ & $3(4.2)$ & $1(1.5)$ & 0.194 \\
\hline Pseudomonas aeruginosa & $1(0.7)$ & $1(1.4)$ & $0(0.0)$ & - \\
\hline Escherichia coli & $13(10.0)$ & $5(7.0)$ & $8(12.1)$ & $\leq \mathbf{0 . 0 0 1}$ \\
\hline Total MDR isolates & 137 & 71 & 66 & \\
\hline
\end{tabular}

Note: $p$ values in bold indicate significant effects.

most predominant in Africa. Gram-positive bacteria were more likely seen as causative organism in paediatric BSI in high-income countries [29]. This variation could be due to epidemiological differences of causative organisms. Staphylococcus aureus (13.2\%) was the second most common isolated organism in the Gram-positive category followed by Streptococcus spp. (4.9\%).

Klebsiella spp. (13.4\%) was the predominant organism isolated among Gram-negative bacteria, followed by Escherichia coli (8.9\%), Pseudomonas spp. (12.5\%), Acinetobacter spp. (1.5\%), Pseudomonas aeruginosa (1.1\%), and Salmonella spp. $(0.8 \%)$. It is interesting to note that Salmonella spp. constitute a prominent pathogen in BSI in studies carried out in the South East Asia region, Bangladesh $[30,31]$, Nepal [32, 33], India [34], and Pakistan [35], but in this study it is not significant. This decrease comparatively could be due to improved urban water management and public health in Maldives. This variation may be due to difference in geographical location and endemic variation. When comparing the significant antimicrobial resistance trends, it can be seen that Enterobacteriaceae isolates demonstrated high resistance to ampicillin and gentamicin as well as second- and third-generation cephalosporins.

Cephalosporins have been used in many settings as empirical therapy [36] mainly due to their low toxicity, broad spectrum activity, and high effectiveness. In this setting, the previous antimicrobial usage and clinical data for the cases have not been evaluated alongside resistance trends. However, the antimicrobial resistance patterns seen here are significant in light of Class $C$ cephalosporinases (Amp C), cabapenemases (including metallo-beta lactamases), and extended spectrum $\beta$-lactamases (ESBL) being the main mechanism for cephalosporin resistance [37]. Further studies with phenotyping are required to generate antibiogram for Gram-positive and Gram-negative isolates. In addition to this, evaluating the extent of resistant isolates can help mitigate the impact of antimicrobial resistance in healthcare settings.

The main limitation of this study, as it was retrospective in nature, was the inability to determine standardization of techniques for individual cases. The other limitation is that comprehensive clinical data and recent antibiotic usage has not been documented. Also, as this study was performed in a single center, it cannot be generalized for the whole population of Maldives.

\section{Conclusions}

In conclusion, this study provides a baseline insight into the bacterial aetiology of bloodstream infections both in paediatric and adult populations. Gram-positive organisms are the major contributors to bloodstream infections in this study. Nevertheless, Gram-negative organisms demonstrated a high percentage of antimicrobial resistance, which needs to be further elucidated. In addition to this, we hope this study would help researchers and policymakers to prioritize respective research options in light of global challenges in combatting antimicrobial resistance.

\section{Abbreviations}

BSI: Bloodstream infections

DIC: Disseminated intravascular coagulation

CLSI: Clinical and Laboratory Standards Institute

CoNS: Coagulase-negative Staphylococcus

MDR: Multidrug resistance.

\section{Data Availability}

The datasets used and/or analyzed during the current study are available from the corresponding author on reasonable request.

\section{Ethical Approval}

The research was ethically approved by the Maldives National University and the National Health Research Committee, Ministry of Health, Maldives. All patient data anonymity was maintained and kept confidential throughout the study.

\section{Conflicts of Interest}

The authors declare that they have no conflicts of interest.

\section{Acknowledgments}

The authors would like to acknowledge the contributions given to this study by Ibrahim Adam, Shazla Mohamed $(\mathrm{PhD})$, and Zeeniya Kamil (PhD). They would like to thank the management of Indhira Gandhi Memorial Hospital and especially the dedicated Medical Laboratory Professionals of 
Diagnostic Laboratory who were involved in data acquisition and entry. This study was funded by the Maldives National University Research Grants, Research Development Office (Reference no. URG-2017-L-AS05).

\section{References}

[1] C. Viscoli, "Bloodstream infections: the peak of the iceberg," Virulence, vol. 7, no. 3, pp. 248-251, 2016.

[2] N. Vasudeva, P. S. Nirwan, and P. Shrivastava, "Bloodstream infections and antimicrobial sensitivity patterns in a tertiary care hospital of India," Therapeutic Advances in Infectious Disease, vol. 3, no. 5, pp. 119-127, 2016.

[3] K. B. Laupland, "Incidence of bloodstream infection: a review of population-based studies," Clinical Microbiology and Infection, vol. 19, no. 6, pp. 492-500, 2013.

[4] H. Lochan, V. Pillay, C. Bamford, J. Nuttall, and B. Eley, "Bloodstream infections at a tertiary level paediatric hospital in South Africa," BMC Infectious Diseases, vol. 17, no. 1, pp. 1-9, 2017.

[5] R. A. Balk, "Severe sepsis and septic shock: definitions, epidemiology, and clinical manifestations," Critical Care Clinics, vol. 16, no. 2, pp. 179-192, 2000.

[6] J. Deen, L. von Seidlein, F. Andersen, N. Elle, N. J. White, and Y. Lubell, "Community-acquired bacterial bloodstream infections in developing countries in south and southeast Asia: a systematic review," The Lancet Infectious Diseases, vol. 12, no. 6, pp. 480-487, 2012.

[7] D. J. Diekema, S. E. Beekmann, K. C. Chapin, K. A. Morel, E. Munson, and G. V. Doern, "Epidemiology and outcome of nosocomial and community-onset bloodstream infection," Journal of Clinical Microbiology, vol. 41, no. 8, pp. 3655-3660, 2003.

[8] A. K. Aung, M. J. Skinner, F. J. Lee, and A. C. Cheng, "Changing epidemiology of bloodstream infection pathogens over time in adult non-specialty patients at an Australian tertiary hospital," Communicable Diseases Intelligence Quarterly Report, vol. 36, no. 4, 2012.

[9] A. J. Kallen, A. I. Hidron, J. Patel, and A. Srinivasan, "Multidrug resistance among gram-negative pathogens that caused healthcare-associated infections reported to the national healthcare safety network, 2006-2008," Infection Control and Hospital Epidemiology, vol. 31, no. 5, pp. 528-531, 2010.

[10] N. R. Payne, J. H. Carpenter, G. J. Badger, J. D. Horbar, and J. Rogowski, "Marginal increase in cost and excess length of stay associated with nosocomial bloodstream infections in surviving very low birth weight infants," Pediatrics, vol. 114, no. 2, pp. 348-355, 2004.

[11] M. Wagner, J. Bonhoeffer, T. O. Erb et al., "Prospective study on central venous line associated bloodstream infections," Archives of Diseases in Childhood, vol. 96, no. 9, pp. 827-831, 2011.

[12] S. N. Hocevar, J. R. Edwards, T. C. Horan, G. C. Morrell, M. Iwamoto, and F. C. Lessa, "Device-associated Infections among neonatal intensive care unit patients: incidence and associated pathogens reported to the national healthcare safety network, 2006-2008," Infection Control and Hospital Epidemiology, vol. 33, no. 12, pp. 1200-1206, 2012.

[13] S. Shrestha, R. Amatya, R. Shrestha, and R. Shrestha, "Frequency of blood culture isolates and their antibiogram in a teaching hospital," Journal of Nepal Medical Association, vol. 52, no. 193, pp. 692-697, 2014.
[14] U. Arora and P. Devi, "Bacterial profile of blood stream infections and antibiotic resistance pattern of isolates," International Journal of Medical Education, vol. 9, no. 4, pp. 186-190, 2007.

[15] S. Moyo, S. Aboud, M. Kasubi, and S. Y. Maselle, "Bacteria isolated from bloodstream infections at a tertiary hospital in Dar es Salaam, Tanzania-antimicrobial resistance of isolates," South African Medical Journal, vol. 100, no. 12, pp. 835-838, 2010.

[16] N. Takeshita, N. Q. Anh, D. M. Phuong et al., "Assessment of bacteremia in a large tertiary care hospital in northern vietnam: a single-center retrospective surveillance study," Japanese Journal of Infectious Diseases, vol. 72, no. 2, pp. 118-120, 2019.

[17] N. P. Parajuli, H. Parajuli, R. Pandit, J. Shakya, and P. R. Khanal, "Evaluating the trends of bloodstream infections among pediatric and adult patients at a teaparajuli," Canadian Journal of Infectious Diseasesa and Medical Microbiology, vol. 2017, Article ID 8763135, 10 pages, 2017.

[18] S. Khurana, N. Bhardwaj, M. Kumari, R. Malhotra, and P. Mathur, "Prevalence, etiology, and antibiotic resistance profiles of bacterial bloodstream infections in a tertiary care hospital in Northern India: a 4-year study," Journal of Lab Physicians, vol. 10, no. 4, pp. 426-431, 2018.

[19] A. Dramowski, M. F. Cotton, H. Rabie, and A. Whitelaw, "Trends in paediatric bloodstream infections at a South African referral hospital," BMC Pediatrics, vol. 15, no. 1, pp. 1-11, 2015.

[20] J. M. Easow, N. M. Joseph, B. A. Dhungel, B. Chapagain, and P. G. Shivananda, "Blood stream infections among febrile patients attending a teaching hospital in western region of Nepal," Australasian Medical Journal, vol. 3, no. 10, pp. 633-637, 2010.

[21] R. MacGregor and H. N. Beaty, "Evaluation of positive blood cultures: guidelines for early differentiation of contaminated from valid positive cultures," Archives of Internal Medicine, vol. 130, no. 1, pp. 84-87, 1972.

[22] M. S. Chang, K. W. Sung, and Y. J. Kim, "Clinical characteristics of bacteremia in children with cancer," Korean Journal of Pediatric Infectious Diseases, vol. 18, no. 2, pp. 201-206, 2011.

[23] S. Chun, C. Kang, Y. Kim, and N. Lee, "Medicina clinical significance of isolates known to be blood culture contaminants in pediatric patients," Medicina (Kaunas, Lithuania), vol. 55, no. 10, p. 698, 2019.

[24] S. S. Richter, S. E. Beekmann, J. L. Croco, D. J. Diekema, M. A. Pfaller, and G. V. Doem, "Minimizing the workup of blood culture contaminants: implementation and evaluation of a laboratory-based algorithm downloaded from," Journal of Clinical Microbiology, vol. 40, no. 7, pp. 2437-2444, 2002.

[25] S. Osaki, K. Kikuchi, Y. Moritoki et al., "Distinguishing coagulase-negative Staphylococcus bacteremia from contamination using blood-culture positive bottle detection pattern and time to positivity," Journal of Infection and Chemotherapy, vol. 26, 2020.

[26] W. Mahavanakul and E. Nickerson, "Feasibility of modified surviving sepsis campaign guidelines in a resource-restricted setting based on a cohort study of severe S. aureus sepsis," PLoS One, vol. 7, no. 2, Article ID e29858, 2012.

[27] L. Liu, S. Oza, D. Hogan et al., "Global, regional, and national causes of child mortality in 2000-2013, with projections to inform post-2015 priorities: an updated systematic analysis," The Lancet, vol. 385, no. 9966, pp. 430-440, 2015. 
[28] N. Droz, Y. Hsia, S. Ellis, A. Dramowski, M. Sharland, and R. Basmaci, "Bacterial pathogens and resistance causing community acquired paediatric bloodstream infections in low- and middle-income countries: a systematic review and meta-analysis," Antimicrobial Resistance and Infection Control, vol. 8, no. 1, 2019.

[29] N. Buetti, A. Atkinson, L. Kottanattu, J. Bielicki, J. Marschall, and A. Kronenberg, "Patterns and trends of pediatric bloodstream infections: a 7 year surveillance study," European Journal of Clinical Microbiology Infection Diseases, vol. 36, no. 3, pp. 537-544, 2017.

[30] W. A. Brooks, A. Hossain, D. Goswami et al., "Bacteremic typhoid fever in children in an urban slum, Bangladesh," Emerging Infectious Diseases, vol. 11, no. 2, pp. 326-329, 2005.

[31] A. Naheed, P. Ram, W. Brooks et al., "Burden of typhoid and paratyphoid fever in a densely populated urban community, Dhaka, Bangladesh," International Journal of Infectious Diseases, vol. 14, no. 3, pp. e93-e99, 2010.

[32] N. Sharma, S. Peacock, W. Phumratanaprapin, N. Day, N. White, and S. Pukrittayakamee, "A hospital-based study of bloodstream infections in febrile patients in Dhulikhel Hospital Kathmandu University Teaching Hospital, Nepal," Southeast Asian Journal of Tropical Medicine and Public Health, vol. 37, no. 2, pp. 351-356, 2006.

[33] S. Pandey, S. Raza, and C. P. Bhatta, "The aetiology of the bloodstream infections in the patients who presented to a tertiary care teaching hospital in kathmandu, Nepal," Journal of Clinical and Diagnostic Research, vol. 7, no. 4, pp. 638-641, 2013.

[34] S. Gupta and B. Kashyap, "Bacteriological profile and antibiogram of blood culture isolates from a tertiary care hospital of North India," Tropical Journal of Medical Research, vol. 19, no. 2, p. 94, 2016.

[35] M. Qureshi and F. Aziz, "Prevalence of microbial isolates in blood cultures and their antimicrobial susceptibility profiles," Biomedica, vol. 27, pp. 136-139, 2011.

[36] T. Hautala, H. Syrjala, V. Lehtinen et al., "Blood culture Gram stain and clinical categorization based empirical antimicrobial therapy of bloodstream infection," International Journal of Antimicrobial Agents, vol. 25, no. 4, pp. 329-333, 2005.

[37] K. Bush, "Bench-to-bedside review: the role of $\beta$-lactamases in antibiotic-resistant gram-negative infections," Critical Care, vol. 14, no. 3, 2010. 